It may be said at once that the enthusiasm with which the scheme was received by educational authorities almost throughout Great Britain has been remarkable, and about two-thirds of the counties of England and Wales have been organised locally by directors of education. As the accompanying map (Fig. 2) shows, large areas have been completed, and testimony is unanimous as to the educational value of the work.

In the second place, there is the permanent value of such a survey. It must be said at the outset that the Land Utilisation Survey is an entirely independent body and has no connexion with any government department or with any political party, and that the inception of the scheme at a time when land valuation was a subject for Parliamentary discussion was purely accidental. Despite the rapidity with which changes in agricultural practice in Great Britain are taking place -for example, there is now less than half the area under the plough that there was fifty years agoit is a fact that no record is available as to the exact areas over which the changes are happening. Statistics are available on a county basis and, in manuscript, on a parish basis, but in the absence of cartographical record it is impossible to evaluate the relative importance of the factors which are causing the change in a given locality. In some areas it may be soil, in others climate. It may be elevation or aspect, accessibility, or merely the progressiveness or lack of progressiveness, as the case may be, on the part of the farmer that has determined the changes in local agricultural practice. It is certain that, if a record of this character can be made for the whole of Great Britain, agricultural economists in the future will have an important basis on which to work.

It has been urged that the volunteer observers lack the training with which to make sufficiently accurate observations. To this there are several answers. In the first place, the scheme is a simple one, and on all hands where there are difficult points the advice of the farmers themselves is being sought and given willingly. In the second place, there is a ready check, in that separate sheets of the six-inch map by different surveyors must fit along the edges where they join, and if there is a disparity in observation, this is at once apparent. In the third place, trained observers are checking the results as they are obtained by making traverses across the sheets.

The map reproduced as Fig. 2 shows the present state of the work. Two things deserve emphasis. First, thanks are due to those who have so nobly assisted up to the present date, when more than four thousand sheets have been completed and returned to the central office; but there are still gaps in the scheme, and in a number of cases the organisation of a whole county is a matter of most urgent necessity. Broadly speaking, it is found that there must be at least one really keen person on the spot for the work to be successful. It is obvious also that any serious delay in the completion of remaining tracts will cause work already done to be vitiated to a considerable extent; for, to be of value, the record should be complete for the years 1931 and 1932. In many of the areas shown on the map as in charge of volunteers there is the need for additional volunteers to fill in remaining tracts. It may be said that the maps are supplied at a reduced rate of $1 s .6 d$. each to those volunteers who wish to keep them when completed. They are merely forwarded to the central office and there copied by a photostat process. The maps themselves are retained at the central office if the surveyor does not wish to purchase them.

The Survey's headquarters are at 18 Houghton Street, W.C.2, and are open daily from 10 to 5, and inquiries are welcomed, by letter and in person. The secretary is Mr. E. C. Willatts, and the advisory committee of the Survey includes representatives from the London School of Economics (in the person of Sir William Beveridge), the Ordnance Survey, the Land Agents' Society, the County Councils Association, the Boy Scouts' Association, whilst agriculturists are represented by the director of Rothamsted Experimental Station (Sir John Russell).

1 Geographical J., 78, July 1931.

Scottish Geographical Mag., 47, May 1931.

\title{
Vortex Motion in Vibrating Columns of Air
}

$\mathrm{O}^{\mathrm{N}}$ $\mathrm{NE}$ of the most familiar and fascinating experiments in acoustics is that initiated by Kundt in 1866, in which the vibrations of an air column enclosed in a glass tube are revealed by means of a fine powder such as cork or lycopodium. Almost everyone is familiar with the manner in which the dust collects in little heaps at the nodes, thereby providing a simple means of estimating the velocity of sound in the gas enclosed in the tube.

An interesting secondary phenomenon also is the formation of a series of ridges or striations of dust lying across the axis of the tube. An attempt to explain this peculiar formation was made in 1891 by W. König. His theory, based on attractions and repulsions between spheres in a vibrating fluid, was universally accepted until the present time. Even Rayleigh found nothing in it to criticise.

In two papers recently published,* Prof. E. N. da C. Andrade has thrown fresh light on the subject in a somewhat startling manner. In the older experiments the observations of the striations were always made under static conditions, that is, after the air vibration had ceased, instead of in the dynamic state whilst the air was in vibration. Not only this, but also the method of excitation was not always maintained for a period sufficiently long for the motion to reach a final stable condition, nor was the amplitude of excitation kept uniform. Prof. Andrade remedies all these defects in

* Proc. Roy. Soc., A, 134, pp. 445-470; 1931; and Phil. Trans. Roy. Soc., A, 230, pp. 413-445; 1932 .

No. 3263, VoL. 129] 
technique by the simple, but very effective, expedient of using a vibrating diaphragm driven by the alternating current from a thermionic valve oscillator of controllable frequency and current output. By such means he has demonstrated that even very small cork dust particles are unsuitable as true indicators of vibratory air motion. Instead of behaving as tracing points they act as obstacles which initiate local vortex motion in the vibrating gas. Consequently, smoke particles are used as a means of studying the true movement of the air in a dust-free tube, suitable precautions (water jackets, etc.) being taken to prevent circulations due to convection currents. A series of beautiful photographs reveals regular circulations of air in the tube, the general drift being from antinode to node along the wall, returning up the centre of the tube. The resonant air column is divided into half wave-lengths by the regular drift-motion of the gas. The form of the stream lines agrees fairly well with Rayleigh's approximate theory.

An entirely novel and most important application of the air vibrations in the resonant tube is made by Prof. Andrade when he utilises the phenomenon to investigate vortex motion around obstacles in the vibrating air. By means of a specially designed tube, and making use of tobacco smoke (from a " cheap cigarette") to indicate the fluid flow, a series of photographs has been taken to show the formation of vortices around objects of simple geometrical form-spheres and cylinders of various diameters. The conditions of formation of the vortices are studied, in particular the critical velocity necessary to initiate vortex motion. In order to determine this velocity it was necessary to measure the actual amplitude of the air vibration, observing the individual smoke particles by means of a microscope. This measurement is in itself a notable achievement, forming as it does the basis of a standard source of sound. The results obtained for the critical velocity in alternating flow are, considering the difficulty of the experiment, in good agreement with similar measurements by other observers for steady flow.

The second of Prof. Andrade's papers deals more particularly with the general behaviour and grouping of solid particles under the influence of air vibrations in tubes. The phenomena observed, antinodal dises, primary and secondary striations, are shown in a most convincing manner to be due to vortex motion and general circulation set up when the air-particle velocity in the tube exceeds the critical value. König's theory is conclusively proved to be untenable as it entirely ignores such vortex motion. It would require too much space to enter into details of the wonderful arrangements of the irregular particles of cork dust, the spherical particles of carnauba wax and the vortex streams around obstructing particles lying in the tube. Actual photographs of the motion of minute dust particles demonstrate the alternating motion superposed on the general drift.

Acoustics has assuredly taken a new lease of life in recent years. The two papers by Prof. Andrade briefly summarised in the foregoing remarks show in a most striking manner how even an ordinary textbook experiment in sound may be revivified to yield most interesting and valuable information. The old Kundt's tube experiment now provides a means not only of measuring sound velocity, but also of producing vibrations of known amplitude (a standard source of sound), and of studying vortex motion. One cannot help wondering, however, how many "cheap cigarettes" Prof. Andrade must have smoked " in the cause of science".

\section{Neanderthal Man in Palestine}

A CABLEGRAM has been received from $\mathrm{Mr}$. Theodore McCown, who, in the temporary absence of Miss Dorothy Garrod, is directing the exploration of caves in Mount Carmel, announcing the discovery of fossil remains of three individuals of the Neanderthal species of mankind. The discovery thus announced is of more than usual importance; it brings to a successful issue a search which has been conducted in Palestine since 1925 , first by the British School of Archæology and latterly by a combined expedition fitted out by that School in conjunction with the American School of Prehistoric Research. Miss Garrod is in charge of the combined expedition, and to her must go the chief credit.

To appreciate the importance of the discovery just announced, it is necessary to recall the sequence of events which have led up to it. The first discovery was made by Mr. Turville Petre in 1925 when excavating a cave on the western shores of Lake Galilee; deep in a Mousterian stratum he found the front part of a skull showing Neanderthal characteristics, but also certain other traits which raised a suspicion that the Neanderthalians of Palestine were racially different from their contemporaries of Europe. In 1928, Miss Garrod, while excavating a cave (Shukbah) on the western flanks of the Judean Hill, penetrated a Mousterian stratum and found a tooth and fragment of skull, which, although undoubtedly Neanderthal in their characterisation, left the racial problem of the ancient Palestinians unsolved. In 1929, Miss Garrod began the excavation of a vast cave in the Wady-el-Mughara in the western flank of Mount Carmel, and again reached a Mousterian stratum.

Although fossil traces were again found in that year and in the following (1930), they were insufficient to throw light on racial characterisation. In 1931, however, the search was crowned with a partial success. Mr. McCown, a student of the American School of Prehistoric Research-recommended by its director, Prof. MacCurdy-joined Miss Garrod, and was placed in charge of the excavation of an adjacent rock-shelter. In the terrace of this shelter, six feet below its surface and four feet within a Mousterian stratum, he exposed 